\title{
Memory for noun pairs in the same or different underlying strings*
}

\author{
KATHRYN C. ALLISON $\dagger$, SLATER E. NEWMAN, and WILLIAM S. FORD \\ North Carolina State University, Raleigh, North Carolina 27607
}

\begin{abstract}
A number of studies have shown that children learn noun pairs faster when they have been presented in a noun-verb-noun context than when they are presented in a noun-conjunction-noun context. Suzuki and Rohwer (1969) hypothesized that the underlying strings of sentences correspond to memory units, making it easier to recall two nouns from the same underlying string than from two different underlying strings. They reported that data from fifth graders supported this hypothesis but that data from college students did not. The experiments reported here were done to determine whether the hypothesis would be supported (a) if the test rate were faster than that used by Suzuki and Rohwer or (b) if sentences longer and more complex than those used by them were employed. The recall data accorded with some predictions derived from the hypothesis. However, data from Ss' posttraining reports on how they learned the pairs suggest that Ss, particularly adults, of ten made up their own strings rather than using those provided, and raise the question of the adequacy of Suzuki and Rohwer's procedure for testing this hypothesis, particularly in adults.
\end{abstract}

A number of experiments (Rohwer, 1967; Rohwer \& Lynch, 1967) have shown that two nouns connected by a verb are learned faster as a pair than are two nouns connected by a conjunction. To account for this "verb facilitation effect," Suzuki and Rohwer (1969) proposed the deep-structure hypothesis, which asserts that the underlying strings of sentences correspond to the functional units of memory storage. Thus, two nouns in the same memory unit (e.g., when connected by a verb) will be learned more easily than two nouns in different memory units (e.g., when connected by a conjunction) because of a simpler retrieval process.

In an experiment to evaluate this explanation, Suzuki and Rohwer (1969) used both simple and complex strings. On study trials, Ss saw each noun pair capitalized and underlined in a syntactical context; on test trials, the first noun of each pair was presented alone and $S$ was instructed to provide the missing noun. Suzuki and Rohwer reported that with both simple and complex strings, fifth graders performed better when the two nouns were connected by a verb (same underlying string condition) than when connected by a conjunction (different underlying string condition), thus supporting the hypothesis. For college students, however, Ss in the

*Experiment I was carried out by W.S.F. for his senior seminar project, and Experiment II is based on an MS thesis by the senior author (Allison, 1971). We appreciate the help of Marian Hamilton, principal of Frances Lacy Elementary School in Raleigh, for arranging for the testing of the sixth-grade Ss in Experiment II, of Dr. Walter E. Meyers, Department of English, North Carolina State University, for assistance in constructing grammatically acceptable and unambiguous subordinate clause sentences for use in Experiment II, and of Drs. Nancy Suzuki and William D. Rohwer, Jr. for making available to us the strings used in their experiment.

$\uparrow$ Requests for reprints should be sent to Kathryn C. Allison, 135 Elmdale Avenue, Akron, Ohio 44313. same and different underlying string conditions performed equivalently.

Suzuki and Rohwer (1969) proposed two explanations for these findings. The first, emphasizing age-related differences in storage, is that adults, but not children, are capable of storing entire sentences as units so that the two nouns appear in the same unit in both kinds of sentences. The second, emphasizing age-related differences in retrieval, is that "the functional storage units for both children and adults are in the form of underlying strings, but that older subjects may require less time to reconstruct the original sentences and thus perform equally well under both conditions when the two nouns are in the same unit and when they are in separate units [Suzuki \& Rohwer, 1969, p. 918]." The present experiments evaluated these positions, Experiment I through manipulating test rate (as suggested by Suzuki and Rohwer), and Experiment II through manipulating complexity and length of the strings. In both experiments, the procedure was similar to that used by Suzuki and Rohwer (1969).

\section{EXPERIMENT I}

In this experiment, adult Ss were exposed to simple or complex strings for which the two nouns were joined by a conjunction or a verb. Within each of these four treatments, half of the Ss were tested at a 4-sec rate, as in the Suzuki and Rohwer experiment, and the rest were tested at a 2-sec rate. According to the storage explanation, adults are capable of storing the entire string, so that the two nouns appear in the same underlying unit for both kinds of strings. Thus, no difference is expected between the same and different underlying string conditions at either a 2 - or a 4 -sec rate.

The retrieval position proposes that for adult Ss, $4 \mathrm{sec}$ 
Table 1

Mean Correct on Each Trial for Each Treatment: Experiment I

\begin{tabular}{|c|c|c|c|c|c|}
\hline \multicolumn{3}{|c|}{ Treatments } & \multirow{2}{*}{\multicolumn{2}{|c|}{ Trials }} & \multirow[b]{3}{*}{ Total } \\
\hline \multirow{2}{*}{$\begin{array}{l}\text { Test } \\
\text { Rate }\end{array}$} & \multirow{2}{*}{$\begin{array}{c}\text { String } \\
\text { Complexity }\end{array}$} & \multirow{2}{*}{$\begin{array}{l}\text { Underlying } \\
\text { String }\end{array}$} & & & \\
\hline & & & 1 & 2 & \\
\hline 2 & Simple & Same & 4.7 & 10.8 & 15.5 \\
\hline 2 & Simple & Different & 6.3 & 12.0 & 18.3 \\
\hline 2 & Complex & Same & 4.4 & 9.9 & 14.3 \\
\hline 2 & Complex & Different & 4.4 & 8.5 & 12.9 \\
\hline 4 & Simple & Same & 8.3 & 14.8 & 23.1 \\
\hline 4 & Simple & Different & 7.3 & 12.5 & 19.8 \\
\hline 4 & Complex & Same & 7.3 & 13.9 & 21.2 \\
\hline 4 & Complex & Different & 3.8 & 9.4 & 13.2 \\
\hline
\end{tabular}

is long enough for retrieval whether the two nouns are in the same or in different underlying strings, even though it may take less time for retrieval under the same condition; $2 \mathrm{sec}$, however, may not be long enough. Thus, an interaction might be expected, no difference between the same and different underlying string conditions occurring when the test rate is $4 \mathrm{sec}$ (replicating Suzuki and Rohwer) but the verb facilitation effect occurring at the $2-\sec$ rate.

\section{Method}

\section{Design and Materials}

The design was a 2 by 2 by 2 factorial with two underlying string conditions (same and different), two test rates ( $2 \mathrm{sec}$ and $4 \mathrm{sec}$, the rate used by Suzuki and Rohwer), and two string types (simple and complex). The simple strings were of the form article noun verb (or conjunction) article noun (e.g., The $B O Y$ and/hit the FLOOR), and the complex strings were of the form article noun verb (or conjunction) article noun conjunction (or verb) pronoun (e.g., The $B O Y$ and/hit the FLOOR hit/and him). The materials were those used in the same four conditions of the Suzuki and Rohwer experiment. There were 24 strings in each list.

\section{Subjects}

Eighty male undergraduates at North Carolina State University served as Ss. They were assigned to the eight treatments in a counterbalanced order. All were tested individually.

\section{Procedure}

The procedure was similar to that used by Suzuki and Rohwer. S was shown one example of the string type for his group and was instructed to read it to himself and to learn the two nouns which were capitalized and underlined, so that later when he saw one of them he could give the other. He was then shown the corresponding test example.

The strings were presented during the two study trials on a memory drum at a $4-\mathrm{sec}$ rate. $E$ read the string aloud while $S$ presumably read it to himself as instructed. On the test trials, each of the first nouns was presented at either a 2- or a 4-sec rate, during which $S$ was to respond orally with the second noun. A different order of items was used on each of the two study and two test trials. During the 2-min interval between the first study trial and the first test, $E$ read aloud the instructions for the test. Subsequent intervals separating study and test trials were each $45 \mathrm{sec}$.

\section{Results and Discussion}

The dependent variable was the number of correct responses on each trial. The mean for each group on each trial is shown in Table 1.

An analysis of variance applied to these data showed the following effects to be significant: test rate, $F(1,72)$ $=7.30, p<.01 ;$ string type, $F(1,72)=6.26, p<.05$; trials, $F(1,72)=40.33, p<.01$; and the Test Rate by Underlying String interaction, $F(1,72)=4.42, p<.05$.

Examination of the means showed that $S$ s did better when the test rate was $4 \mathrm{sec}$ than when it was $2 \mathrm{sec}$, and that, as with Suzuki and Rohwer's adults, there were more correct responses for simple than for complex strings.

The significant Underlying String by Test Rate interaction derived from better performance on the same than on different underlying strings at the 4-sec rate (thus, failing to replicate the results of Suzuki and Rohwer); there were, however, no differences in performance on the same and different strings at the 2-sec rate.

The results of this experiment provide support neither for the storage nor for the retrieval positions. Contrary to the storage position, the verb facilitation effect was found to be dependent on test rate; and contrary to the retrieval position, the verb facilitation effect was observed at $4 \mathrm{sec}$ but not at $2 \mathrm{sec}$, whereas the reverse had been predicted. These two positions were evaluated in a different way in Experiment II.

\section{EXPERIMENT II}

In this experiment, the storage and retrieval explanations were compared through use of sentences longer and more complex than those used by Suzuki and Rohwer. The simple strings were the same as those used by Suzuki and Rohwer, but the complex strings were subordinate clause sentences of the form, "The boy caught the egg as he hit the floor." This has two underlying strings: "The boy caught the egg," and "The boy hit the floor." In this example, the two nouns to be learned, "boy" and "floor," are in the same underlying string. However, when the surface structure is changed slightly to make "The boy caught the egg as it hit the floor," "boy" and "floor" are in different underlying strings ("The boy caught the egg" and "The egg hit the floor").

Both children and adults were used in this experiment. For children, the same results obtained by Suzuki and Rohwer were expected with both the simple and the complex strings. However, for adults, the storage position predicts no differences for simple or complex strings as a function of type of underlying string, whereas the retrieval position predicts a replication of 
Table 2

Mean Correct on Each Trial for Each Treatment: Experiment II

\begin{tabular}{|c|c|c|c|c|c|c|c|}
\hline \multirow[b]{3}{*}{ Age } & \multicolumn{2}{|c|}{ Treatments } & \multirow{2}{*}{\multicolumn{4}{|c|}{ Trials }} & \multirow[b]{3}{*}{ Total } \\
\hline & \multirow{2}{*}{$\begin{array}{c}\text { String } \\
\text { Complexity }\end{array}$} & \multirow{2}{*}{$\begin{array}{c}\text { Underlying } \\
\text { String }\end{array}$} & & & & & \\
\hline & & & 1 & 2 & 3 & 4 & \\
\hline Children & Simple & Same & 3.9 & 9.1 & 10.7 & 11.1 & 34.8 \\
\hline Children & Simple & Different & 4.6 & 8.4 & 9.6 & 9.9 & 32.5 \\
\hline Children & Complex & Same & 2.8 & 5.6 & 7.3 & 8.1 & 23.8 \\
\hline Children & Complex & Different & 4.8 & 8.1 & 9.4 & 10.0 & 32.3 \\
\hline Adults & Simple & Same & 7.7 & 10.7 & 10.9 & 11.7 & 41.0 \\
\hline Adults & Simple & Different & 5.3 & 8.2 & 9.8 & 10.0 & 33.3 \\
\hline Adults & Complex & Same & 6.2 & 8.8 & 10.2 & 10.9 & 36.1 \\
\hline Adults & Complex & Different & 6.1 & 9.4 & 11.2 & 11.7 & 38.4 \\
\hline
\end{tabular}

$M S E$ between $(72)=17.86$

$M S E$ within $(216)=1.63$

Suzuki and Rohwer's results with simple strings, but better performance on complex strings when the two nouns are in the same underlying string.

There were two other important differences from Experiment I, the use of four rather than of two trials and the use of a posttraining interview. Four trials were used to determine whether the difference between underlying string conditions would increase if training were extended. Suzuki and Rohwer had noted that the difference did increase (though not significantly) from the first to the second test, and a similar trend was evident in Experiment I.

In Experiment I, a number of Ss mentioned that in learning the pairs they had made their own strings rather than use those provided by E. In the present experiment, Ss were shown each of the pairs and asked to indicate how they had tried to learn them. This was done to ascertain whether they had actually used the strings whose effects were being investigated.

\section{Method}

\section{Design and Materials}

The design of the experiment was a 2 by 2 by 2 factorial with two ages (sixth grade and college), two string types (simple string and subordinate clause sentences), and two underlying string conditions (same and different). From the noun pairs used by Suzuki and Rohwer (1969), 12 were selected for which unambiguous subordinate clause sentences could be constructed In the simple string conditions, Suzuki and Rohwer's strings were used for the 12 noun pairs chosen. The simple strings were like those used in Experiment I (e.g., The $B O Y$ and/hit the $F L O O R$ ), whereas the complex strings were subordinate clause sentences (e.g., The $B O Y$ caught the egg as it/he hit the FLOOR).

\section{Subjects}

Forty undergraduates at North Carolina State University and 40 sixth-grade children from a middle-class public elementary school served as Ss. In assigning adult Ss to the four treatments, a counterbalanced order was used separately with males and with females. Use of this order with the first seven replications of children (boys and girls combined, however. rather than separated) led to differences in the number of boys and girls in each treatment. For the remaining Ss (five boys and seven girls), no more than two were assigned consecutively to the same treatment. There were 10 of each age group, five males and five females, in each treatment. All Ss were tested individually.

\section{Correct Responses}

The main dependent variable was the number of correct responses on each trial. The mean for each group on each trial is presented in Table 2 . An analysis of variance was applied to these data.

In the between-Ss part of the analysis, only age $[F(1,72)=$ $11.29, \mathrm{p}<.01]$ and the String Type by Underlying String interaction $[F(1,72)=7.57, p<.01]$ were significant. As in the Suzuki and Rohwer experiment, adults gave more correct responses than did children. The significant interaction of String Type by Underlying String derived from better recall for the same underlying string condition when simple strings were used and for the different underlying string condition when subordinate clause sentences were used.

It was anticipated that for children, the deep-structure hypothesis would be supported with both the simple and complex strings. For adults, however, the storage position predicted no support for the deep-structure hypothesis with either simple or complex strings, whereas the retrieval position predicted support for the deep-structure hypothesis with complex strings but not with simple strings. Although the interaction of Age by Underlying String by String Type was not significant, individual comparisons were done to test the above predictions. The results of these comparisons showed that in the simple strings, the mean for the same condition was significantly higher than in the different condition for adults but not for children, whereas for the subordinate clause sentences, the mean for the different condition was significantly higher for children but not for adults. Thus, neither position was supported.

In the within-Ss part of the analysis, in addition to the main effect of trials $[F(3,216)=273.01, p<.001]$, two interactions were significant, Age by Trials $[F(3,216)=2.88, p<.05]$ and Age by Underlying String by Trials $[\mathrm{F}(3,216)=2.76, \mathrm{p}<.05]$. Examination of the means revealed that (1) the difference between adults and children was greatest on Trial 1 and remained approximately the same on Trials $2-4$, and (2) the difference between underlying string conditions tended to narrow as training proceeded for both adults and children; for adults, the smallest difference (in favor of the same condition) occurred on Trial 3, whereas for children, the smallest difference (in favor of the different condition) occurred on Trial 4.

\section{Interview Data}

of the 40 children, 30 reported one or more methods for learning the pairs. The dominant method was (a) using the 
strings provided $(\mathrm{N}=12)$, followed by (b) making up their own strings $(\mathrm{N}=11)$, or $(\mathrm{c})$ repeating the nouns to be remembered $(\mathrm{N}$ $=7$ ). Only $2 \mathrm{Ss}$ in these latter two groups reported using the strings provided to remember one or more of the pairs. The children reported using the strings provided for $35.1 \%$ of the pairs, making up their own strings for $38.8 \%$, and repeating the nouns for the remaining $26.1 \%$.

For adults, the results were different. Of the 40 adults, 39 reported making up their own strings to remember half or more of the pairs. However, 10 of these Ss did report using the strings provided by $E$ to remember at least one of the pairs. Adult Ss reported making up their own strings for $94.9 \%$ of the pairs, using the strings provided by $\mathrm{E}$ for $2.8 \%$, and repeating the nouns for the remaining $2.3 \%$ of the pairs.

In the simple string conditions, children in the same string groups (who performed slightly but not significantly better than children in the different string group) reported most often using the strings provided by $\mathrm{E}$ to learn the pairs, whereas in the different string groups, Ss reported more frequently using their own strings or repeating the noun pairs. For the complex strings, the differences in reported strategies were less marked. Ss in the same string group (who performed more poorly than Ss in the different string group) reported slightly more frequent use of the strings provided by $\mathrm{E}$ than of their own strings, whereas for $\mathrm{Ss}$ in the different string group, the reverse occurred. For all four of the adult treatment groups, Ss were much more likely to report making up their own strings than using either of the other two strategies.

\section{Discussion}

The results from this experiment do not appear to provide support for either the storage or the retrieval positions. Contrary to predictions derived from both positions, evidence apparently in support of the deep-structure hypothesis occurred for adults with the simple, though not with the complex strings, while for children, the deep-structure hypothesis appeared to be supported with neither simple nor complex strings. (It was, in fact, surprising to find that with the complex strings, children did better when the two nouns were in different, rather than in the same, underlying strings.) Though the results for adults exposed to simple strings replicated those of Experiment I, neither these results nor those for children exposed to simple strings replicated the findings of Suzuki and Rohwer.

The data from the posttraining interviews suggest that this technique did not provide an adequate test of the deep-structure hypothesis. Neither tha children nor the adults in Experiment II reported using the strings provided by $\mathrm{E}$ in learning all of the pairs. It appears, from the interview data, that adults are more likely than children to make up their own strings rather than use those provided by $\mathrm{E}$.

Since Ss in Experiment I were adults, and since adults often make up their own strings, it may be that the deep-structure hypothesis was not adequately tested in that experiment either. In a similar study, Duffy and Montague (1971) have shown that their adult Ss reported making up their own strings for $36.8 \%$ of the pairs presented in complex strings like those used in Experiment I. On the basis of their findings and those of Experiment II, it seems possible that other experiments studying the verb facilitation effect in adult Ss (e.g., Suzuki, 1972; Suzuki \& Rohwer, 1969) may also not have provided an adequate test of the deep-structure hypothesis, since Ss in these experiments (unbeknownst to $\mathrm{E}$, who did not ask questions about the strategies used by these Ss) may have made up their own strings for some of the pairs rather than use those provided by $\mathrm{E}$.

The results of Experiment II and of Duffy and Montague (1971) emphasize the need for insuring that the strings provided by $\mathrm{E}$ are those used by $\mathrm{S}$ whether a child or an adult. They emphasize also the importance of determining, perhaps through posttraining interviews or through requiring posttraining free recall of the entire strings, whether or not $\mathbf{S}$ has done so.

\section{REFERENCES}

Allison, K. C. Memory for noun-pairs in the same or different underlying strings: A test of the deep structure hypothesis. Unpublished MS thesis, North Carolina State University, 1971.

Duffy, T. M., \& Montague, W. E. Sentence mnemonics and noun pair learning. Journal of Verbal Learning \& Verbal Behavior, $1971,10,157-162$.

Rohwer, W. D., Jr. Social class differences in the role of linguistic structures in paired-associate learning. Final report, November 1967, University of California, Berkeley, Contract OE-6-10-273, Project 5-0605, Office of Education.

Rohwer, W. D., \& Lynch, S. Form class and intralist similarity in paired-associate learning. Journal of Verbal Learning \& Verbal Behavior, 1967, 6, 551-554.

Suzuki, N. Noun-pair learning in children and adults: Underlying strings and retrieval time. Child Development, 1972, 43, 299-307.

Suzuki, N., \& Rohwer, W. D., Jr. Deep structure in the noun-pair learning of children and adults. Child Development, 1969, 40, 911-919.

(Received for publication October 9, 1972; revision received December 27,1972 .) 